Ireneusz Stolarczyk

The Pontifical University of John Paul II in Cracow, Poland

\title{
Die Familie angesichts der Migration im Lichte der Botschaften von Johannes Paul II. zum Welttag der Migranten
}

\author{
The Family in View of the Migration \\ in the Light of Messages of John Paul II for the World Day of Migrants
}

Abstract

The author of this article presents migration as a social phenomenon, which constitutes a challenge for both the international community and particular countries. The involvement of the Holy Father John Paul II, as well as the variety of problems featuring in messages for the World Day of Migrants allow the reader to perceive the issues connected with migration as extremely urgent - both for individuals and for communities, among which the family is of greatest importance. The reason why the Holy Father committed himself to this field was to uphold human dignity and a global social solidarity. The analysis of this phenomenon allows the Holy Father to address a call for responsibility to various groups whose integral activity can make the phenomenon of migration more dignified. The messages of the Holy Father also offer a direct invitation to all people of goodwill to make their own contribution so that every person and family are respected and discriminations that undertime human dignity.

\section{Keywords}

Migration, family, human dignity, Church, state. 
Die Migration, als gesellschaftliches Phänomen, stellt eine Herausforderung für die internationale Gemeinschaft und die einzelnen Staaten ${ }^{1}$ dar. Aus diesem Grund hat sie auch im Interessenkreis der katholischen Soziallehre ihren Platz gefunden. Die Vielfalt der menschlichen Erfahrungen, die mit diesem Prozess zusammenhängen, ist so groß, dass sie in der Soziallehre der Kirche oft und sehr konkret behandelt werden. Einerseits treten hier Themen auf, die mit dem Recht auf Leben auf dem Gebiet des eigenen Landes verbunden sind, andererseits wird aber das Recht betont, sein eigenes Land zu verlassen und sich in einem anderen Staat niederzulassen. Die Grundlage für dieses Recht ist die Tatsache, dass dieselbe Person sowohl ein Bürger eines bestimmten Landes als auch der ganzen Welt ist. Die Soziallehre der Kirche betont, dass es notwendig ist, den Emigranten das Recht auf Emigration zuzuerkennen - welche die günstige Integration fördert und den Emigranten den beruflichen Aufstieg und den Erhalt einer guten Wohnung erleichtert. Gleichzeitig kritisiert die Soziallehre der Kirche enge Nationalismen, welche die wahre Brüderlichkeit - die Grundlage des wirklichen Friedens und der wirklichen Gerechtigkeit - unmöglich machen. Der einzige Grund, die Migration einzuschränken, ist das gemeinsame Wohl des Staates, der die Immigration oder Emigration erlebt.

Das Engagement des Heiligen Vaters Johannes Pauls II. und der Reichtum der Themen, die die Botschaften zum Welttag der Migranten enthalten, lassen die mit der Migration verbundenen Probleme als wichtig und außergewöhnlich aktuell - sowohl für die einzelnen Menschen als auch für die von ihnen gebildeten Gemeinschaften, von denen die Familie eine der wichtigsten ist - betrachten. In diesen Gemeinschaften kommen auch viele grundsätzliche Thesen der päpstlichen Soziallehre zum Ausdruck. Der Grund dafür, dass der Heilige Vater dieses Thema aufgenommen hat, ist die Verteidigung der Menschenwürde und der damit verbundenen allgemeingesellschaftlichen Solidarität. Die Analyse der Fragen dieses Phänomens des gesellschaftlichen Lebens gibt dem Heiligen Vater die Möglichkeit, einen Appell an verschiedene Menschengruppen zu richten, deren integrierte Wirkung das Migrationsphänomen des Menschen und der Familie, in der er lebt, würdiger machen kann. Es ist der Appell an die Kirche, die für den Zustand und die Stärke des Glaubens der Migranten verantwortlich ist; es ist der Appell an die Staaten, die für die Rechtssetzung sowie für die Bildung der gesellschaftlichen Strukturen und der Gesellschaftsordnung Verantwortung tragen $^{2}$, es ist auch der Appell an die Migranten selbst, die sich für ihre

${ }^{1}$ Vgl. Jan Paweł II, Nie wolno łamać ani ignorować praw migranta. Orędzie Ojca Świętego na światowy Dzień Migranta 1996, „L’Osservatore Romano” 6 (1996), S. 8.

${ }^{2}$ Vgl. M. Zając, Emigracja wyzwaniem duszpasterskim dla Kościoła, Lublin 2009, S. 62. 
Familien verantwortlich fühlen und auch für die gesellschaftlich-wirtschaftliche Entwicklung ihres Vaterlandes verantwortlich sind. Die Botschaften des Heiligen Vaters drücken die Überzeugung aus, dass die schwere Lage der Migranten und deren Familien nicht die Folge des unabänderlichen Schicksals ist, sondern dass sie sich dank der verantwortungsvollen Entschlüsse der Menschen guten Willens als menschlicher erweisen kann.

Dem Migrationsphänomen hat der Papst Johannes Paul II. in seiner Lehre viel Platz geschenkt. Besonders gut bekannt ist die päpstliche Lehre, die die Botschaften enthält, welche an die Gesellschaften zum Migrantentag gerichtet werden. Eben der Inhalt dieser Botschaften wird zum Gegenstand der vorliegenden Überlegungen. Wenn man die Frage nach dem Schicksal der Familien in der Migrationszeit stellt, muss man die Beurteilung dieses Phänomens in Bezug auf die Würde der Familie und deren Rolle in der Bildung jeder Gemeinschaft in Betracht ziehen sowie - dem ersten Teil der Überlegungen entsprechend - die Gefahren zeigen, welche mit dem Funktionieren der Familie unter den Migrationsbedingungen zusammenhängen und die vom Autor der Botschaften vorgeschlagenen Lösungen darstellen.

\section{Die Würde der Familie als Kriterium für die Bewertung der Migration}

Das Interesse des Papstes für die komplizierte Situation der Migranten ist im Kontext der gesamten Soziallehre von Johannes Paul II. zu betrachten. Im Vordergrund der päpstlichen Lehre steht immer der Mensch in der Fülle seiner natürlichen und übernatürlichen Würde. Es ist hier daran zu erinnern, dass sich die in der päpstlichen Lehre als spezifischer Wert der menschlichen Person gezeigte Würde des Menschen auf das Bestehen der Person bezieht und unter zwei Aspekten - dem natürlichen und dem übernatürlichen - betrachtet wird. Die erste Dimension drückt die Fähigkeit des Menschen aus, frei und bewusst zu wirken. In der Lehre des Heiligen Vaters wird diese Wahrheit durch das Betonen der Größe des Menschen in Bezug auf die Freiheit und Vernünftigkeit der menschlichen Natur ausgedrückt. Die andere Dimension der Würde enthüllt die Teilnahme des Menschen an der transzendenten Wirklichkeit, am Leben Gottes selbst. Der Papst weist darauf hin, dass die menschliche Würde ein unreduzierbarer Wert ist. Von der Würde des Menschen leitet er auch die Wahrheit ab, dass der Mensch die Pflicht hat, sich zu vervollkommnen ${ }^{3}$ und sich in der Sphäre der höchsten

\footnotetext{
${ }^{3}$ Vgl. Jan Paweł II, Orędzie Ojca Świętego na Światowy Dzień Migranta 1992, „L'Osservatore Romano" 10 (1992), S. 6.
} 
Güter selbst zu verwirklichen. Das alles verursacht, dass man den Menschen den gesellschaftlichen Strukturen, der Ökonomie oder irgendwelchen materiellen Gütern nicht unterordnen kann.

Die päpstliche Lehre über die Würde der einzelnen Personen verbindet sich mit der Wahrheit über die Würde der Gemeinschaft, die die Familie darstellt, weil ihre Rolle für die Erhaltung der Würde des empfangenen und geborenen Lebens unersetzlich ist. Diese Wahrheiten werden in den Botschaften zum Migrantentag ausgedrückt. Aus Rücksicht auf den unwiederholbaren Wert des Menschen finden in den päpstlichen Botschaften Appelle zum Bruch mit der gegenständlichen Behandlung der Migranten Platz. Das verbindet sich mit der Anerkennung ihres Rechts auf Arbeit, auf kulturelle Identität und auf Verbindung mit dem eigenen Volk und hängt auch mit der vollständigen Entwicklungsmöglichkeit zusammen ${ }^{4}$. Der Papst sieht das Bedürfnis, eine Ordnung für den Umgang mit Migranten zu schaffen, die durch die Anerkennung aller ihnen gebührenden Rechte ihre volle persönliche und berufliche Selbstverwirklichung - zumal die ganze Familie oder deren Teil in der Migration ist - ermöglichen könnte. Die Staaten, in denen das Phänomen der Migration zum gesellschaftlichen Problem wird, werden aufgefordert, es menschenwürdig zu lösen ${ }^{5}$.

Aus dem Respekt vor der Menschenwürde wegen ihrer übernatürlichen Dimension, ergeben sich die Hinweise des Papstes, die das Recht der einzelnen Migranten und deren Familien, ihren Glauben zu bekennen, betreffen. Diese Dimension des unwiederholbaren Wertes des Menschen verlangt, der Familie die Möglichkeit zu sichern, den Glauben in ihrer Muttersprache zu bekennen und die Seelsorge unter den Migranten wie auch einen entsprechenden religiösen Dialog - wenn der Migrant vor der Glaubenskonfrontation steht - zu führen. Das Unmöglichmachen, den Glauben zu bekennen, und der eigenartige religiöse Synkretismus, der einen vortrefflichen Grund für die Tätigkeit der Sekten ist ${ }^{6}$, stellt häufig ein großes Problem für die ganzen Migrantenfamilien dar. Zweifellos steht das in Widerspruch zu der Achtung vor der Menschenwürde in deren übernatürlichen Dimension?

${ }^{4}$ Vgl. H. Skorowski, Imigracja a prawa człowieka, in: J. Balicki (Hrsg.), Migracja jako nowa kwestia społeczna, Katowice 2003, S. 23.

${ }^{5}$ Vgl. Jan Paweł II, Orędzie Ojca Świętego na Światowy Dzień Migranta 1991, „L’Osservatore Romano" 9-10 (1991), S. 38.

${ }^{6}$ Vgl. Jan Paweł II, Niebezpieczeństwo prozelityzmu sekt religijnych. Papieskie Orędzie na Światowy Dzień Migracji 1990, „L'Osservatore Romano” 7-8 (1990), S. 8.

${ }^{7}$ Vgl. P. Kaczmarczyk, Migracje zarobkowe Polaków w dobie przemian, Warszawa 2005, S. 48 . 
Aus der in der Lehre von Johannes Paul II. betonten Menschenwürde ergibt sich als deren Folge - die zwischenmenschliche Solidarität. Als moralische Einstellung ist sie die feste und beständige Entschlossenheit, sich für das Gemeinwohl einzusetzen, das heißt für das Wohl aller und eines jeden ${ }^{8}$. In seiner Lehre betont der Papst die Verbindung der Solidarität mit der menschlichen Natur und dadurch mit der Würde. Der Solidarität liegt die Einheit des Menschengeschlechts zugrunde. Sie wächst unter dem Einfluss des gemeinsamen Schicksalsgefühls auf. Das ist nicht ohne Bedeutung für die Migranten, ihre Familien und die Gesellschaften, in denen sie leben. Es ist zu bemerken, dass mit der Hochschulbildung und dem immer größeren Interesse für die Kultur anderer Völker das Solidaritätsgefühl mit der Familie und das Streben nach Einheit auch heutzutage zunimmt. Es nimmt ebenfalls die Empfindlichkeit für die Würde der menschlichen Person und deren unveräußerlichen Rechte - insbesondere derjenigen, die mit dem Funktionieren der Familie zusammenhängen - sowie das Verantwortungsgefühl für internationale Fragen zu. Der Heilige Vater betont die - für die Migration positiven Erscheinungen, deren Autoren verschiedene Menschengruppen sind: Staatsbeamte, Gelehrte, Kaufleute, Techniker, Unternehmer und auch für die Erweiterung der Kultur und Information handelnde Personen. Bemerkenswert sind auch Organisationen von internationalem Charakter und Kultureinrichtungen, die den jungen migrierenden Familienangehörigen die Möglichkeit bieten, zwischen verschiedenen Wegen der weiteren inneren Entwicklung unter den Universitäten vieler Länder ${ }^{9} \mathrm{zu}$ wählen.

Die Solidarität der Menschen der Welt gewinnt besonderen Charakter im Kontext der Glaubensgemeinschaft. Während den vorstehenden Überlegungen über die Solidarität der Menschen der Welt die natürlichen Voraussetzungen zugrunde lagen, findet die Einheit der Menschen - die sich aus der Tatsache ergibt, dass Gott die Menschen als sein Abbild geschaffen hat - in dieser Berufung eine neue Motivierung, eine neue Empfindlichkeit. Das ermöglicht dem modernen Menschen, alle Diskriminierungsformen zu überschreiten, erinnert ihn an die Gleichheit aller Völker und veranlasst zum zusätzlichen Respekt vor der menschlichen Person - dem Mitbruder im Glauben ${ }^{10}$. In dieser Weise - wie das der Heilige Vater betont - betrifft der Glaube nicht nur das persönliche Leben jedes

\footnotetext{
${ }^{8}$ Vgl. Jan Paweł II, Sollicitudo rei socialis, 38.

${ }^{9}$ Vgl. Jan Paweł II, Orędzie Ojca Świętego na Światowy Dzień Migranta 1991, „L'Osservatore Romano" 9-10 (1991), S. 37.

${ }^{10}$ Vgl. R. Dzwonkowski, Jan Pawet a emigracja i Polonia, Lublin 1991, S. 64.
} 
Menschen, sondern drückt sich im Einsatz für die Nächsten und deren Familien aus ${ }^{11}$.

Die Solidarität die sich aus der Brüderlichkeit in Christus und der Zugehörigkeit zur Familie Gottes ergibt, ist eine Herausforderung für die Christen, sich für die Lösung der Probleme der Migration einsetzen. Darauf deutete Johannes Paul II. hin: Solidarität bedeutet, gegenüber dem, der in Schwierigkeiten ist, Verantwortung wahrzunehmen. Für den Christen ist der Migrant nicht einfach ein Individuum, das er nach gesetzlich festgelegten Vorschriften zu respektieren hat, sondern eine Person, deren Anwesenheit ihn herausfordert und deren Bedürfnisse seine Verantwortung verpflichten. „Wo ist dein Bruder?“ (Gen. 4,9) Die Antwort ist nicht in den vom Gesetz vorgeschriebenen Grenzen, sondern im Stil der Solidarität zu geben ${ }^{12}$.

\section{Die Gefahren auf den Migrationswegen der Familien}

Die Jahresbotschaften von Johannes Paul II. zum Welttag der Migranten enthalten eine Analyse der Probleme, auf die der Migrant und seine Familie stoßen. Die vom Papst dargestellten Probleme, mit denen die Migranten ringen müssen, sind sehr vielfältig. Um die vorliegende Analyse klar zu machen, kann man sie in einige allgemeine Gruppen einteilen. Zu der ersten zählen diejenigen, welche die integrale Entwicklung des Migranten und seiner Familie erschweren oder unmöglich machen ${ }^{13}$. Das widerspricht unmittelbar der These über den Respekt vor der menschlichen Würde.

Der Heilige Vater deutet auf das schwere Schicksal der Migranten hin und analysiert diese Situation unter dem Aspekt des Leidens, der Not und der Unsicherheit. Die Migrationen, die vielmals aus ökonomischen Gründen unternommen werden, stoßen auf Widerwillen der entwickelten Länder. Die Stratifikation und Struktur der Länder, in denen die Immigranten ankommen, ermöglichen nur in beschränktem Maße den Ankömmlingen die Anpassung und setzen dadurch sie und ihre Familien den Problemen aus, die sie selbst nicht lösen können. Die kritische Stimme des Papstes betrifft vor allem die Situationen, in denen den Migrantenmengen nur die einfachsten Beschäftigungen - welche die

${ }^{11}$ Vgl. Jan Paweł II, Katolicy Świeccy a emigracja. Papieskie Orędzie na Światowy Dzień Emigracji, „L'Osservatore Romano” 9-10 (1987), S. 3.

${ }^{12} \mathrm{Vgl}$. Jan Paweł II, Nie wolno łamać ani ignorować praw migranta. Orędzie Ojca Świętego na Światowy Dzień Migranta 1996, „L'Osservatore Romano” 6 (1996), S. 8.

${ }^{13}$ Vgl. R. Dzwonkowski, Jan Pawet a emigracja i Polonia, S. 82. 
größte Mühe verlangen und am schlechtesten bezahlt werden - zur Verfügung stehen. In dieser Situation sind die Migranten mit ihren ganzen Familien immer die ärmsten in der Perspektive ihrer Akzeptierung, ihrer Sicherheit, ihrer Rechte und ihrer Chancen auf den gesellschaftlichen und beruflichen Aufstieg. Der Heilige Vater bemerkt, dass es das wiederum erschwert, eine Arbeit und eine Wohnung zu finden, und einen negativen Einfluss auf die Möglichkeit, an den Hochschulen zu studieren, ausübt ${ }^{14}$.

Eines der Hauptthemen, die Johannes Paul II. in seinen Botschaften zum Welttag des Migranten behandelt, ist die Frage der Arbeit ${ }^{15}$. Der Papst bemerkt, dass die Migration eben wegen der Arbeitsprobleme oft zur Verletzung der menschlichen Würde führt und die dem Menschen zustehenden Rechte wegnimmt. Nur so kann man die mit der Suche nach Arbeit, die den jungen Männern und Frauen Pseudoagenturen anbieten, zusammenhängende Migration betrachten. Die Menschen, welche im Land ihrer Träume ankommen, müssen sich mit den Schwierigkeiten und Hindernissen auseinandersetzen, von denen sie nichts gewusst haben oder die ihnen als leicht zu überwältigen dargestellt wurden ${ }^{16}$. Das zieht weitere Probleme nach sich, die mit einer langwierigen Arbeitslosigkeit oder der Aufnahme von schlecht bezahlten Beschäftigungen verbunden sind. Johannes Paul II. nennt diese Situation ,neuer Sklavenhandel ${ }^{\text {“17 }}$. Die Familie, die kein Einkommen hat, kann ihre Grundfunktionen nicht ausüben.

Die integrale Entwicklung des Menschen gründet auf der Befriedigung der Bedürfnisse materieller und geistiger Natur. Unter den geistigen Bedürfnissen weist der Papst auf diese hin, die sich auf das übernatürliche Leben beziehen. Die Migrationssituation erweckt auch hier begründete Befürchtungen ${ }^{18}$. Der Heilige Vater bemerkt, das die Sekten und die neuen Religionsbewegungen ihre Aktivität auf die Migranten und deren Familien konzentrieren. Ihre schwere gesellschaftliche Situation, Instabilität und Trennung von der Familienkultur bilden sehr gute Bedingungen für die Arbeit der Sekten, die aggressive und aufdringliche Methoden anwenden. Es lässt sich in dieser Situation nicht verschweigen, dass die Migranten, oft herausgerissen aus dem gesellschaftlichen Leben ihres Herkunftslandes, alieniert aus der Gesellschaft, in der sie sich

\footnotetext{
${ }^{14}$ Vgl. Jan Paweł II, Papieskie Orędzia na Światowy Dzień Migranta 1989, „L'Osservatore Romano" 9 (1989), S. 4.

${ }^{15}$ Vgl. B. Andrees, Praca przymusowa jako forma handlu, in: Handel ludźmi - zapobieganie i ściganie, Z. Lasocik (Hrsg.), Warszawa 2006, S. 198.

${ }^{16}$ Vgl. Jan Paweł II, Problem uchodźców. Orędzie na Światowy Dzień Emigranta, „L'Osservatore Romano" 8 (1984), S. 28.

${ }^{17}$ Ibidem, S. 28.

${ }^{18} \mathrm{Vgl}$. B. Andrees, Praca przymusowa jako forma handlu, S. 200.
} 
niedergelassen haben, oft gezwungen zum Leben außerhalb der objektiven, ihre Rechte schützenden Ordnung, mit dem Verzicht auf ihren eigenen Glauben dafür bezahlen, dass sie Hilfe brauchen und nicht am Rande der Gemeinschaft leben wollen ${ }^{19}$. Dieser Preis tut der menschlichen Würde Abbruch und ist ein Schlag für die Familienstabilität.

Die nächste Gruppe von Problemen, die Papst Johannes Paul II. in der Wirklichkeit des Lebens der Migranten bemerkt, sind Erschwerungen im Familienleben, wenn ein Elternteil ein Migrant ist, oder wenn die ganze Familie die Unsicherheit des Lebens der Migranten erlebt. Als Paradox stellt Johannes Paul II. die Tatsache dar, dass sich die Eheleute aus Sorge für das Wohl der Familie zum Migrantenschicksal entschließen, wodurch sie sich selbst und auch ihre Familien vielen Schwierigkeiten und schweren Proben aussetzen. Es gehören dazu lang dauernde Trennung, welche den Eheleuten die Pflichten auferlegt, die sie bisher nicht gewohnt waren, Trennung der Eltern von den Kindern, Unsicherheit und Instabilität, Erschwerungen in der Bildung der Kinder, elende Wohnungen in schmutzigen Wohnvierteln der großen Metropolen, zu hohe Arbeitsstundenzahl, niedrige Löhne, die die harmonische und normale Entwicklung der Familie erschweren, und keine Möglichkeit, den Kindern die Muttersprache und vaterländische Kultur zu vermitteln, was verursacht, dass sich die Kinder von den Eltern entfernen ${ }^{20}$. Die Situation, in der die Kinder von anderen Werten als denen ihrer Eltern leben - und manchmal von solchen, die zu den durch die Eltern anerkannten Werten in Widerspruch stehen - ist die Ursache für weiter Dramen im Familienkreis. An diesen Problemen fehlt es auch in den Mischehen nicht ${ }^{21}$.

Die Schwierigkeiten, mit denen die Familie in der Emigration ringen muss, bildeten eine Grundlage für den hübschen Vergleich ihres Schicksals mit dem der Heiligen Jungfrau. Johannes Paul II. sieht im Leben Mariä die Vorwegnahme und die Widerspiegelung des Lebens der Migranten: Außerdem erlebte Maria die Mühe der Vertreibung und Emigration auf dem fremden Boden (...). Das war eine unerwartete Flucht, die plötzlich in der Nacht unternommen wurde. Diese Flucht war von der euch, den Emigranten, so gut bekannten Pein und Unruhe begleitet: von dem Schmerz wegen der Trennung von den Personen und von dem, was man liebt, von der Aufgabe der euch so lieben Hoffnungen, von der Wanderung über

${ }^{19}$ Vgl. Jan Paweł II, Niebezpieczeństwo prozelityzmu sekt religijnych. Papieskie Orędzie na Światowy Dzień Migracji 1990, „L'Osservatore Romano” 7-8 (1990), S. 8.

${ }^{20}$ Vgl. Jan Paweł II, Matżeństwo i rodzina. Papieskie Orędzie na Światowy Dzień Emigracji, „L'Osservatore Romano” 9 (1986), S. 22.

${ }^{21}$ Vgl. Ibidem, S. 24. 
unbekannte Orte, von den Schwierigkeiten bei der Suche nach einer Unterkunft in der Fremde, wo alles unbekannt ist, von der Unsicherheit, ob es gelingt, eine Arbeit zu finden, die den Lebensunterhalt sichert, von der Atmosphäre der Diskriminierung und des Misstrauens, die man häufig in der Umgebung fühlt, vom Provisorium der Situation, in der alle Lebenspläne - sowohl die eigenen als auch die der Familienangehörigen und besonders diejenigen, welche die Kinder betreffen - unsicher $\operatorname{sind}^{22}$.

\section{Vorschläge der Hilfswirkungen für die migrierende Familie}

Die Botschaften zum Welttag der Migranten enthalten eine tiefgründige Analyse ihres Lebens. Die Botschaft des Heiligen Vaters schließt aber nicht mit der Darstellung des Sachverhalts ab. Aus der Analyse der Wirklichkeit leitet der Papst konkrete Belehrungen ab, die er an verschiedene Gruppen - auch an die migrierende Familiengemeinschaften - richtet. Die synthetische Analyse des Inhalts der Botschaften lässt bemerken, dass Johannes Paul II. die Lösung der schweren Situation der Migranten vom übereinstimmenden Zusammenwirken der drei Gruppen: der Kirche, des Staates und der migrierenden Gesellschaften, erwartet. Das bringt den Appell der Enzyklika Rerum novarum in Erinnerung, in der sich Leon XIII. an ähnliche Gruppen wandte und eine schnelle Lösung der schwierigen Arbeiterfrage erwartete ${ }^{23}$.

Der Heilige Vater weist darauf hin, dass der Immigrant-Katholik überall dort, wo er sich mit seiner Familie niederlässt, ein Mitglied der Lokalkirche wird. So hat er alle ihm zustehenden Rechte und Pflichten. Der Papst deutet darauf hin, dass die Weise, in der er von dieser Kirche empfangen wird, ein Zeugnis und Prüfstein für die Katholizität ist. Die uneigennützige Sorge und das Verständnis sind oft die wertvollsten Anzeichen des Wohlwollens, das der Immigrant in einer schweren und unbekannten Situation erlebt. So drückt sich die Sorge des Erlösers selbst aus, der mit der Botschaft der Liebe gekommen ist und eben diese Botschaft in seiner Kirche als Testament hinterlassen hat. Die christliche Gemeinschaft darf beim Empfang der Migranten - wegen einer Störung bei der Erfüllung ihrer täglichen Pflichten - keine Unzufriedenheit zeigen. Der Papst erwartet die

${ }^{22}$ Vgl. Jan Paweł II, Papieskie Orędzie na Światowy Dzień Emigranta, „L'Osservatore Romano” 10-11 (1988), S. 32.

${ }^{23}$ Vgl. Leon XIII, Rerum novarum, Teil. II. 
Atmosphäre der Freude und Akzeptierung ${ }^{24}$. Diese Atmosphäre, die häufig über die ökonomischen, politischen oder kulturellen Einteilungen hinausgeht, ergibt sich aus der Tatsache, dass jeder Mensch von Gott geliebt wird und potentiell von Christus erlöst ist; dadurch ist er in dem gleichen Maß des Respekts, der Liebe, des Dienstes und der Betreuung würdig ${ }^{25}$.

Eine unbestreitbare Aufgabe der Kirche gegenüber der Migranten ist die seelsorgerische Betreuung, welche alle die Erhaltung und Vertiefung des Glaubens bestrebenden Wirkungen umfasst ${ }^{26}$. Diesem Ziel dient die Erwachsenenkatechese, derEinsatzderMigranten imaktiven liturgischenLeben derGlaubensgemeinschaft, die Beteiligung an der Sakramentsspendung, angefangen mit der Taufe, die Bildung der Gebetsgemeinschaften, wodurch das Zeugnis von der Liebe gegeben wird. Das ist eine Chance, die Glaubenseinheit in der Verschiedenartigkeit des Migrantenlebens zu schaffen ${ }^{27}$. Nach der Meinung des Heiligen Vaters soll diese Aufgabe vor allem von der Fachseelsorge in den verschiedenen Ländern, die für die Familien Fürsorge trägt, aufgenommen werden. Eben von ihnen erwartet der Migrant die Hilfe nicht nur bei der Vertiefung des Glaubens, sondern auch bei der Kontaktaufnahme mit dem Milieu, bei der Annahme einer neuen, manchmal einer ganz fremden Kultur ${ }^{28}$, beim Lösen der Probleme der Mischehen ${ }^{29}$ oder der Ehen, die wegen der Migration die Trennung von der Familie erleben ${ }^{30}$.

Die päpstlichen Botschaften betonen auch eine wesentliche Rolle des Staates beim Lösen der Migrationsprobleme. Es ist hier zu betonen, dass diese Pflichten für ihren Betreuungscharakter in den Händen der staatlichen Institutionen liegen. Daher ergeben sie sich direkt aus dem Subsidiaritätsprinzip. Bei der Behandlung der Staatspflichten geht der Heilige Vater auf die Rechtsfragen und Staatsstrukturen ein. Sowohl das Recht als auch die im Rahmen der Staatstätigkeit gebildeten Strukturen haben den Immigranten und ihren Familien die Anpassung

${ }^{24}$ Vgl. Jan Paweł II, Niebezpieczeństwo prozelityzmu sekt religijnych. Papieskie Orędzie na Światowy Dzień Migracji 1990, „L’Osservatore Romano” 7-8 (1990), S. 8.

${ }^{25}$ Vgl. Jan Paweł II, Orędzie Ojca Świętego na Światowy Dzień Migranta 1991, „L’Osservatore Romano" 9-10 (1991), S. 38.

${ }^{26}$ Vgl. R. Dzwonkowski, Jan Pawet a emigracja i Polonia, S. 122.

${ }^{27}$ Vgl. Jan Paweł II, Papieskie Orędzie na Światowy Dzień Migranta 1989, „L’Osservatore Romano" 9 (1989), S. 4.

${ }^{28}$ Vgl. Jan Paweł II, Orędzie Ojca Świętego na Światowy Dzień Migranta 1993, „L'Osservatore Romano" 11 (1993), S. 4.

${ }^{29}$ Vgl. Ibidem, S. 5.

${ }^{30}$ Vgl. Jan Paweł II, Małżeństwo i rodzina. Papieskie Orędzie na Światowy Dzień Emigracji, „L’Osservatore Romano” 9 (1986), S. 22; Jan Paweł II, Problem uchodźców. Orędzie na Światowy Dzień migranta, „L'Osservatore Romano” 8 (1984), S. 28. 
an die neuen Bedingungen, gesellschaftliche Bildung und Orientierung zu sichern, die ihnen hilft, aus der Isolation und dem Unwissen im Gebiet der Rechts-, Sozial-, Erziehungs- und Fürsorgepolitik des sie aufnehmenden Landes herauszukommen ${ }^{31}$.

Nach der päpstlichen Soziallehre wirken sich die Aufgabe dieser Pflichten und die rechtlichen und strukturellen Vernachlässigungen ungünstig auf das gemeinsame Wohl aus. Sie sind ein Hindernis auf dem Weg der solidarischen Entwicklung der Menschheit und erniedrigen die Würde der menschlichen Person - sie tragen zur Verbreitung der Sündenstrukturen bei ${ }^{32}$. Der Rücktritt davon besteht in der Tätigkeit des Staates für die Anerkennung der Immigranten und ihrer Familien als vollberechtigte Mitglieder der Gesellschaft, an deren Schicksal sie teilnehmen und dadurch zum wirtschaftlichen und gesellschaftlichen Fortschritt beitragen ${ }^{33}$. Das ist auch die Sorge für den Wiederaufbau der gesunden Umgebungs- und Gesellschaftsstruktur der vernachlässigten Stadtviertel, in denen die Emigranten häufig wie außerhalb der normalen Gesellschaft leben müssen. Es ist auch für alle klar, dass die Notwendigkeit besteht, jegliche Diskriminierung - unter anderem durch die Lösung des Arbeitslosigkeitsproblems - beim Zugang zu den Wohnungen, den Arbeitsplätzen oder zur Arztversorgung ${ }^{34}$ zu vermeiden. Mit anderen Worten: der Staat hilft den Immigranten durch solche rechtlichen und strukturellen Wirkungen, welche es ihnen ermöglichen, das zu erhalten, was er normalerweise seinen Bürgern und deren Familien sichert. Einerseits ist das eine Verteidigung vor dem Rassismus und allen Versuchen, die Immigranten in den Hintergrund zu drängen, andererseits ist das die Festigung der Kultur der Solidarität, welche den Immigranten die Schaffung der Bedingungen für ein ruhiges menschenwürdiges Leben und für eine menschenwürdige Entwicklung sichert ${ }^{35}$. Die Tätigkeit des Staates strebt dadurch danach, die mit der Wohnung, der Arbeit, der Sicherheit, der anderen Sprache, der Kultur und der Bildung verbundenen Probleme der einzelnen Familienangehörigen unter den Migrationsbedingungen zu lösen.

${ }^{31}$ Vgl. Jan Paweł II, Matzeństwo i rodzina. Papieskie Orędzie na Światowy Dzień Emigracji, „L'Osservatore Romano” 9 (1986), S. 22.

${ }^{32}$ Vgl. Jan Paweł II, Papieskie Orędzie na Światowy Dzień Emigranta, „L'Osservatore Romano” 10-11 (1988), S. 32.

${ }^{33}$ Vgl. B. Andrees, Praca przymusowa jako forma handlu, S. 193.

${ }^{34}$ Vgl. Jan Paweł II, Orędzie Ojca Świętego na Światowy Dzień Migranta 1992, „L'Osservatore Romano" 10 (1992), S. 6.

${ }^{35}$ Vgl. Jan Paweł II, Orędzie Ojca Świętego na Światowy Dzień Migranta 1993, „L'Osservatore Romano" 11 (1993), S. 4. 
In seinen Botschaften richtet der Heilige Vater Johannes Paul II. auch einen Appell an die Migranten selbst. Das ist ein Ausdruck der Sorge um die Verminderung der mit dem Migrationsphänomen zusammenhängenden Probleme. Angenommen, dass die ökonomisch-gesellschaftlichen Gründe die Mehrheit der Migrationsursachen darstellen, appelliert der Papst um die Erhöhung des Lebensniveaus der sich entwickelnden Staaten. Die Gesellschaften dieser Staaten sollen ihre Entwicklung selbst leiten und selbst für die Familie sorgen, wodurch sie die Intensität der Migration vermindern ${ }^{36}$. Der Heilige Vater ermutigt, die selbständigen Initiativen für die Erhöhung des Alphabetisierungsund Grundbildungsniveaus zu ergreifen und weist auf den Nutzen hin, den die Auswertung der natürlichen Vorräte und des menschlichen Potentials sowie die in den Industrieländern erworbene Ausbildung bringt. Diese Tätigkeit ist bestimmt ein Schlüssel zum wirtschaftlichen und gesellschaftlichen Fortschritt der sich entwickelnden Länder und dadurch zur Verminderung des Migrationsphänomens selbst ${ }^{37}$.

\section{Bibliography}

Andrees B., Praca przymusowa jako forma handlu, in: Z. Lasocik (Hrsg.), Handel ludźmi-zapobieganie i ściganie, Warszawa 2006.

Balicki J. (Hrsg.), Migracja jako nowa kwestia społeczna, Katowice 2003.

Bos-Karczewska M., Migracja czy zagrożenie, Gdańsk 2005.

Dzwonkowski R., Jan Pawet a emigracja i Polonia, Lublin 1991.

Kaczmarczyk P., Migracje zarobkowe Polaków w dobie przemian, Warszawa 2005.

\footnotetext{
${ }^{36}$ Vgl. M. Bos-Karczewska, Migracja czy zagrożenie, Gdańsk 2005, S. 47.

${ }^{37}$ Vgl. Jan Paweł II, Orędzie Ojca Świętego na Światowy Dzień Migranta 1992, „L’Osservatore Romano" 10 (1992), S. 6.
} 